\title{
Parasite density and impaired biochemical/hematological status are associated with severe clinical aspects of canine visceral leishmaniasis
}

\author{
Alexandre B. Reis ${ }^{\mathrm{a}, \mathrm{b}, *}$, Olindo A. Martins-Filho ${ }^{\mathrm{c}}$, Andréa Teixeira-Carvalho ${ }^{\mathrm{a}}$, \\ Maria G. Carvalho ${ }^{\mathrm{d}}$, Wilson Mayrink ${ }^{\mathrm{e}}$, João C. França-Silva ${ }^{\mathrm{e}}$, \\ Rodolfo C. Giunchetti ${ }^{a, b}$, Odair Genaro ${ }^{\mathrm{e}}$, Rodrigo Corrêa-Oliveira ${ }^{\mathrm{b}}$ \\ ${ }^{a}$ Laboratório de Parasitologia e Histopatologia, Núcleo de Pesquisas em Ciências Biológicas/NUPEB, Instituto de Ciências Exatas e Biológicas, \\ Universidade Federal de Ouro Preto, Rua Costa Sena, 21 Centro, 35400000 Ouro Preto, Minas Gerais, Brazil \\ ${ }^{\mathrm{b}}$ Laboratório de Imunologia Celular e Molecular, Centro de Pesquisas René Rachou, Fundação Oswaldo Cruz, \\ Av. Augusto de Lima, 1715 - Barro Preto, Belo Horizonte, Minas Gerais, Brazil \\ ${ }^{\mathrm{c}}$ Laboratório de Doença de Chagas, Centro de Pesquisas René Rachou, Fundação Oswaldo Cruz, Belo Horizonte, Minas Gerais, Brazil \\ ${ }^{\mathrm{d}}$ Laboratório de Hematologia Clínica, Faculdade de Farmácia, Universidade Federal de Minas Gerais, Belo Horizonte, Minas Gerais, Brazil \\ e Laboratório de Leishmanioses, Departamento de Parasitologia, Instituto de Ciências Biológicas, \\ Universidade Federal de Minas Gerais, Belo Horizonte, Minas Gerais, Brazil
}

Accepted 14 September 2005

\begin{abstract}
We have performed a detailed investigation in 40 dogs naturally infected with Leishmania infantum (syn. chagasi), subdivided into three groups: asymptomatic $(\mathrm{AD}=12)$, oligosymptomatic $(\mathrm{OD}=12)$ and symptomatic $(\mathrm{SD}=16)$, based on their clinical features. Twenty non-infected dogs (CD) were included as control group. Serological analysis, performed by IFAT and ELISA, demonstrated higher antibodies titers in SD in comparison to the AD. A positive correlation was found between parasite density in the spleen and skin smears as well as the bone marrow parasitism with clinical status of the infection. We observed that the progression of the disease from asymptomatic to symptomatic clinical form was accompanied by intense parasitism in the bone marrow. It is likely that this led to the impaired biochemical/hematological status observed. Finally, we believe that the follow-up of these parameters could be a relevant approach to be used as markers during therapeutic and vaccine evaluations.
\end{abstract}

(C) 2005 Elsevier Ltd. All rights reserved.

Keywords: Canine visceral leishmaniasis; Leishmania infantum (syn. chagasi); Parasite density; Biochemical/hematological status

\section{Introduction}

Visceral leishmaniasis (VL) is one of the most relevant and emergent diseases worldwide, reaching $98 \%$ of mortality in non-treated human cases (Tesh, 1995). Beside its broad epidemiological spectrum, VL is an important zoonosis widely spread in tropical and subtropical areas of the globe. In the last 16 years, 37,294 new human cases of VL were reported in Brazil (Vieira and Coelho, 1998).

\footnotetext{
* Corresponding author. Tel.: +55 313349 7778; fax: +55 3132953115 . E-mail address: alexreis@cpqrr.fiocruz.br (A.B. Reis).
}

From the epidemiological standing point, the canine visceral leishmaniasis (CVL) is considered to be more important than the human disease, due to its higher prevalence and the fact that both asymptomatic and symptomatic dogs are equally infectious to the vectors (Molina et al., 1994). Many asymptomatic animals, in endemic areas, have been detected with parasites in the skin (Marzochi et al., 1985), and from 1980 to 1997 , a total of 414,168 seropositive dogs were identified in Brazil (Vieira and Coelho, 1998).

The CVL may evolve from asymptomatic cases to a systemic disease, which mostly culminates in death. From the 
localized cutaneous infection, the parasite can be disseminated via lymphatic or blood vessels, infecting macrophages of the bone marrow, lymph node, liver and spleen, as well as kidneys and gastrointestinal tract. Initial clinical signs are hypertrophy of lymph node, dermatitis and periorbital and nasal dermatitis that can disseminate. The bristles become opaque and fall, together with onychogriphosis and edema of the paws. Other signs such as fever, apathy, diarrhea, intestinal hemorrhage, weight loss, hepatosplenomegaly, hyperkeratosis, cutaneous ulceration, particularly on the nose, ears, tails, and keratoconjunctivitis are frequent, although not necessarily present in all animals (Genaro et al., 1988; Dias et al., 1999). The CVL presumptive diagnosis is generally performed by serological tests, such as indirect immunofluorescence assay test (IFAT) and enzyme linked immunosorbent assay (ELISA), conjunction with clinical and epidemiological records. The major problem regarding clinical diagnosis is the fact that CVL signs are very similar to those observed in other infectious diseases. The chronic aspect of the disease and its long incubation period may generate a delay or failure in clinical diagnosis (Cardoso and Cabral, 1998). Despite its high sensitivity, serological tests present a broad range of cross-reactions with other protozoan (Costa et al., 1991; Grimaldi and Tesh, 1993). However, the parasitological diagnosis is generally low in sensitivity when parasite density is low.

There are several biochemical and hematological alterations in naturally or experimentally infected dogs. The findings include normocytic/normochromic anemia, and increase of total serum proteins levels. It seems that biochemical alterations are linked to a polyclonal humoral immune response, which leads to raised protein levels in serum (Marzochi et al., 1985). This can be observed by an increase of the total serum proteins with hyperglobulinemia and hypoalbuminemia, with decreased albumin/globulin ratio (Cardoso and Cabral, 1998; Strauss-Ayali and Baneth, 2001). Considering the re-emergent aspect of visceral leishmaniasis, in several parts of the world, the epidemiological importance of dogs, we assessed both the clinical and laboratorial aspects from canine visceral leishmaniasis contributing to monitor the clinical status of dogs during infection.

\section{Materials and methods}

\subsection{Animals}

Sixty mixed breed adult dogs of both genders aging from 2- to 6-years old were selected. They were maintained in the kennel of the Institute of Biological Sciences of Federal University of Minas Gerais or provided by the Center of Zoonosis (Zoonosis Center - Belo Horizonte City Council) of Minas Gerais state, Brazil. The clinical pre-selection was carried out in the latter location. The animals were kept in quarantine with drinking water and a balanced feed $\left(\right.$ Kinus $^{\circledR}$ - BRASWEY - AS) given ad libitum.
The dogs inserted in this study were strayed or domiciled mongrel dogs, selected based on their serological results on IFAT, used as a "gold standard" immunological test for diagnosis of CVL. Animals presenting IFAT titers $\geqslant 1: 40$ were considered positive and included into the infected groups. Animals with IFAT negative at 1:40 were considered non-infected and included as a control group. Leishmania infected dogs did not receive any treatment for CVL and were euthanatized to proceed the organs collections, including skin, spleen, liver and lymph nodes. As chemotherapeutical practices for CVL is not officially allowed in Brazil, all infected dogs must be submitted to euthanasia.

Infection with Leishmania infantum was confirmed in all IFAT positive dogs by at least one additional serological and/or parasitologial approach used.

\subsection{Blood sample collection}

After quarantine, blood samples were collected in 20 c.c. disposable sterile syringes, preferentially from jugular and/ or cephalic veins. Five milliliter-samples were transferred to tubes with EDTA (in the proportion of $1 \mathrm{mg} / \mathrm{ml}$ ) for the hemogram and $5 \mathrm{ml}$ were transferred at a tube with no anticoagulant. The serum samples were stored in aliquots at $-20^{\circ} \mathrm{C}$, until use in the serological and biochemical tests.

\subsection{Clinical and parasitological evaluations}

The dogs were clinically classified, according to presence/absence of infection signs: asymptomatic (AD, $n=12$ ), with no suggestive signs of the disease; oligosymptomatic (OD, $n=12$ ), with maximum three clinical signs including opaque bristles and/or localized alopecia and/ or moderate loss of weight; symptomatic ( $\mathrm{SD}, n=16)$, with characteristic clinical signs of visceral leishmaniasis, such as opaque bristles, severe loss of weight, onychogriphosis, cutaneous lesions, apathy and keratoconjunctivitis; noninfected dogs (CD), animals for negative serological and parasitological exams for Leishmania, considered here as control animals.

The assessment of parasitological parameters was performed by the isolation of the parasite in bone marrow cultures, obtained by aspiration of the bone marrow, in NNN/LIT medium. The cultures were maintained in incubators at $23{ }^{\circ} \mathrm{C}$ and examined three times every 10 days. Bone marrow aspiration was undertaken from the inferior region of the sternum or from the iliac crest, under sedation $(25 \mathrm{mg} / \mathrm{kg}$ live weight of sodium tiopental - Thionembu$\mathrm{tal}^{\circledR}$ - via intravenous). Parasitological diagnoses in tissue smears (ear skin, spleen, liver and popliteal lymph node) were performed after necropsy of the animals. The fragments of the tissues were used in slide smears by apposition of two microscopy slides. The smears were stained by Giemsa and examined under optical microscopy, for the identification of amastigote forms of Leishmania. The par- 
asite density evaluation was performed, in bone marrow, skin and spleen smears and the results expressed as "Leishman Donovan Units" (LDU index), according to Stauber (1955), which correspond to the number of Leishmania amastigote by 1000 nucleated cells. This study was approved by the Ethical Committee for the use of Experimental Animals of the Universidade Federal de Minas Gerais, Brazil (CETEA).

\subsection{Serological assessment}

For the IgG assessment, IFAT, ELISA-extract and ELISA-rK39 were performed. For IFAT, Leishmania amazonensis promastigote (MHOM/BR/1960/BH6) were used. These parasites were maintained in logarithmic growth in LIT medium, as described by Camargo and Rebonato, 1969. For IFAT, specific anti-canine IgG antibodies FITC-conjugated diluted 1:200, as recommended by the manufacturer (Biomanguinhos, FIOCRUZ, RJ, Brazil). Animals with antibody titration higher than 1:40 were considered to be positive. The results are expressed as antibody titers.

For ELISA-extract tests, soluble antigens from $L$. infantum (syn. chagasi) promastigote (MHOM/BR/1972/BH46) were used. The parasites were cultured for 7 days in LIT medium (Mancianti et al., 1995), washed three times by centrifugation at $2000 \mathrm{rpm}$ in phosphate buffer solution (PBS) $\mathrm{pH} \mathrm{7.2,} \mathrm{for} 10 \mathrm{~min}$, followed by three ultra-sound cycles of $1 \mathrm{~min}$ at $40 \mathrm{~W}$ on ice bath (Sonifier Cell Disruptor ${ }^{\circledR}$ - Branson Sonic Power Co., EUA). The sonicated material was centrifuged at $18,500 \mathrm{rpm}$ for $1 \mathrm{~h}$ and 30 min at $4{ }^{\circ} \mathrm{C}$. The supernatant was transferred to dialysis tubes and dialyzed through PBS for $36 \mathrm{~h}$, and submitted to four PBS changes every $6 \mathrm{~h}$. Finally, the remaining material was filtered in disposable sterile filters of $22 \mu \mathrm{m}$ under aseptic conditions; one aliquot was taken for protein dosage by the method of Lowry et al. (1951), and adjusted to the concentration of $1000 \mu \mathrm{g} / \mathrm{ml}$ and stored in small aliquots at $-70{ }^{\circ} \mathrm{C}$ prior to use.

The recombinant antigen rK39, specific for Leishmania of the donovani complex (Badaró et al., 1997) was used.

For ELISA tests, including both ELISA-extract and ELISA-rK39, an anti-canine IgG peroxidase-conjugated was used at 1:8000 dilution (Bethil Laboratories, INC Montgomery Texas, EUA). Sera samples were tested at the dilution of 1:80 and the optical density obtained with an automated ELISA reader (Bio-Rad - 2550, EUA) at $492 \mathrm{~nm}$.

Results from ELISA-extract test were considered positive when optical density was $\geqslant 140$. Data from ELISA $\mathrm{r}-\mathrm{K} 39$ were classified considering the optical density $\geqslant 90$ as the edge between the negative and positive results.

\subsection{Biochemical and hematological evaluation}

Serum proteins were determined by the Biuret reagent and the readings performed in a spectrophotometer in
$510 \mathrm{~nm}$ (CELM E-225D). The electrophoretic profile of the serum proteins was carried out using cellulose acetate strips according to the manufacturer instructions (CELOGEL $^{\circledR}$ ). The electrophoretic readings were performed in a spectrophotometer and analyzed with the software CS93101 PC.

Hemogram was performed by conventional technique of counting erythrocytes and leukocytes (Dace and Lewis, 1984) in an automatic cell counter-CELM CC 510.

\subsection{Statistical analysis}

Statistical analysis was performed using the Minitab 9.2 software package. In the parametric data, one-way analysis of variance (ANOVA) was used for the comparative study between groups. Student's $t$-test was used for determining the differences between the groups' averages. In the nonparametric data, Kruskal-Wallis test was used for the comparative study between groups, followed by Dunn's test. Spearman rank correlation was calculated to compare results of the different methods. The differences were considered significant when the probabilities of equality, $p$ values, were $\leqslant 0.05$.

\section{Results}

\subsection{Serological parameters}

Total IgG reactivity results in infected animal sera as evaluated by IFAT, ELISA-extract and ELISA rk-39 are shown in Fig. 1. Statistical analysis confirmed that uninfected controls selected by presenting negative IFAT, also differ from the infected animals when tested by ELISAextract and ELISA-rK39 serology. The mean ELISA reactivity of all CVL carrier groups was higher than that observed for the control group $(p<0.001)$.

No significant difference was observed ELISA-extract and ELISA rk-39 in total IgG reactivity among the CVL groups. On the other hand, IFAT reactivity revealed that SD displayed a higher serological titer in comparison to AD.

Despite the methodology applied to evaluate the immunological status of infected dogs, a positive correlation was observed between serological reactivity and the clinical status (IFAT $r=0.4207, \quad p=0.0069 ; \quad$ ELISA-extract $r=0.8041, p<0.0001$; ELISA sK39 $r=0.7573, p<0.001$ ).

\subsection{Parasitological parameters}

Parasite detection on different tissues from the dog host is shown in Table 2. Although SD displayed higher frequency of positive results in comparison to $\mathrm{AD}$, regardless the tissue evaluated, statistical analysis demonstrated that only AD and OD displayed differences on the frequency of positive parasitological analysis depending on the tissue specimen. In this context, our data demonstrated lower performance of bone marrow impression smears in $\mathrm{AD}$ 

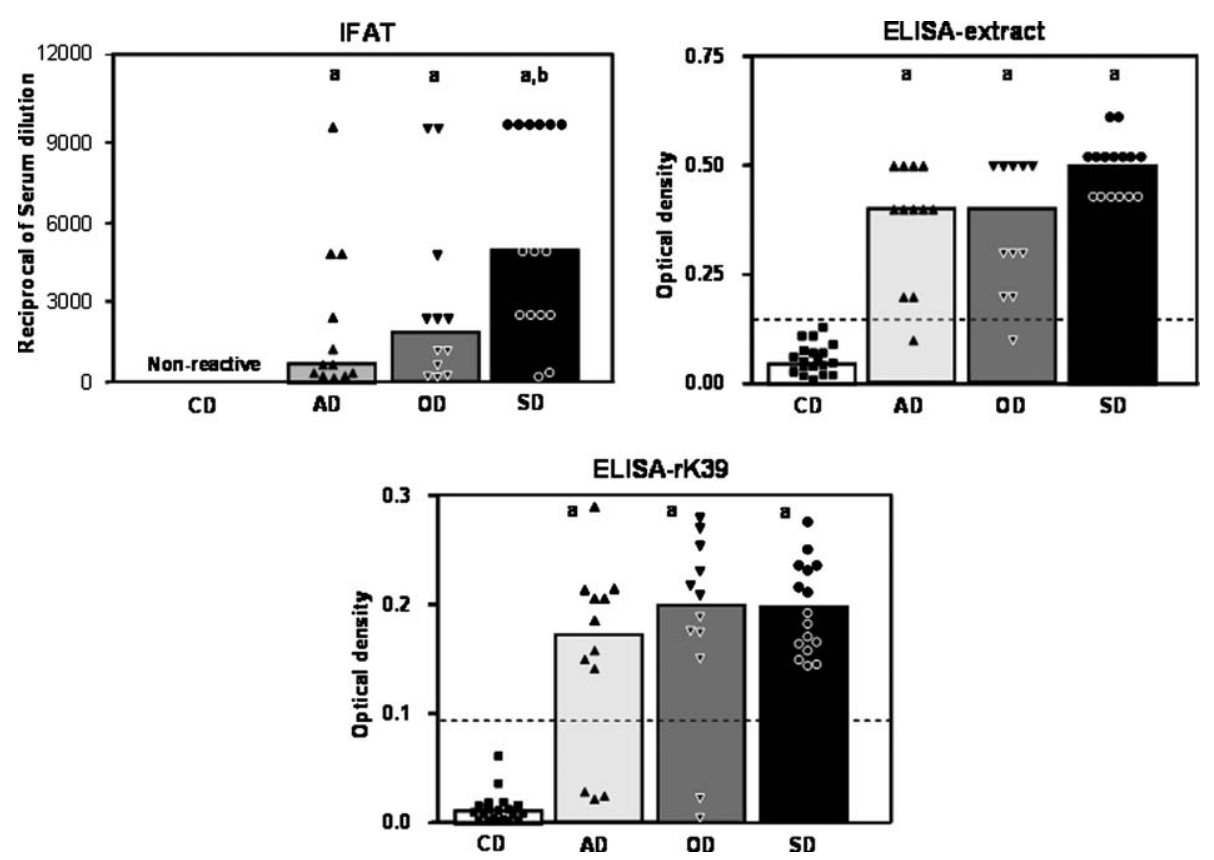

Fig. 1. Reactivity of Total IgG in serum from CVL infected dogs. IFAT results are shown as scattering of individual values and mean serum dilution. ELISA-extract results (cut-off $=140$ ) and ELISA-rK39 (cut-off $=90$ ) are shown as scattering of individual values and mean optical density. The letters "a" and "b" represent statistically significant differences as compared to control $(\mathrm{CD})$ and asymptomatic dogs $(\mathrm{AD})$, respectively. NR $=$ non-reactive.

group, whereas higher sensitivity of skin impression smears was identified for OD in comparison to the other tissues.

Further analysis of parasite load, reported as "Leishman Donovan Units" demonstrated that skin, bone marrow and spleen LDU indexes in AD was lower when compared to SD group. No significant differences in LDU values, including those from the skin, bone marrow and spleen, were observed among the OD and all others infected groups (Fig. 2). When we compared LDU values in the different tissues analyzed we observed that all infected animals display higher skin LDU indexes in comparison to bone marrow. Moreover, our data demonstrated a positive correlation among bone marrow LDU and spleen LDU $(r=0.6188 ; p=0.0319)$ in AD dogs, while a positive correlation was observed between skin LDU and spleen LDU $(r=0.9746 ; p=0.0001)$ in the OD dogs. Only for SD dogs we observed a positive correlation between skin and bone marrow LDU values $(r=0.7994 ; p=0.0002)$.
Correlation analysis of parasite density and serological findings demonstrated that $\mathrm{AD}$ showed a positive correlation between skin, bone marrow and spleen LDU and IFAT (skin $r=0.8304 ; p=0.0008$, bone marrow $r=0.8271 ; p=0.0009$, spleen $r=0.5782 ; p=0.0489$ ).

\subsection{Biochemical and hematological parameters}

The evaluation of biochemical parameters, related to protein alterations, showed no significant difference in total protein levels among all groups. However, a significant decrease $(p<0.001)$ in albumin concentrations, in the oligosymptomatic and symptomatic dogs, was observed, when compared to control and asymptomatic animals (Fig. 3). Dogs of the oligosymptomatic group presented a significant increase in globulin concentration $(p<0.001)$, when compared to control group, while the symptomatic group presented a significant increase $(p<0.001)$ in globu-
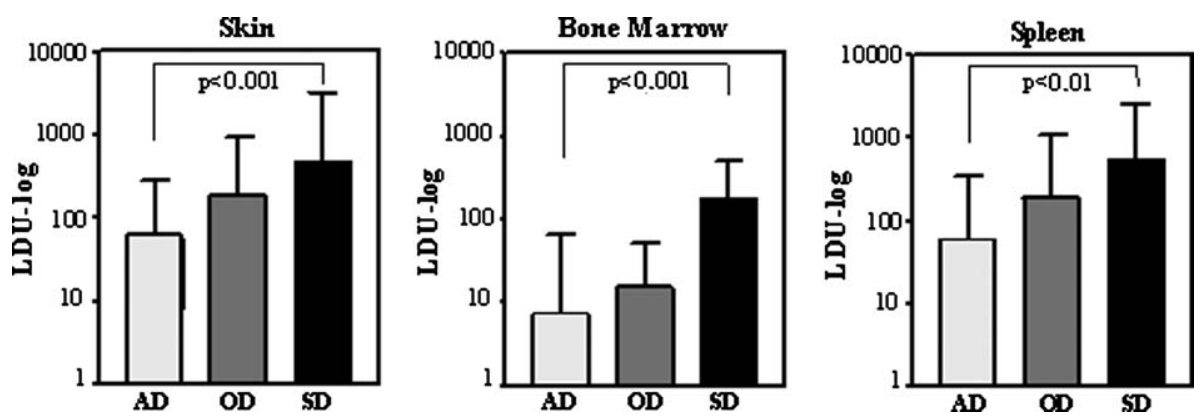

Fig. 2. Index of parasite density determined by LDU in skin, bone marrow and spleen of naturally infected dogs by $L$. infantum. The results are shown as median and maximum/minimum values. Asymptomatic dogs $=$ (unstained bars); oligosymptomatic dogs $=($ gray bars $)$ and symptomatic dogs $=($ black bars). Results are expressed as number of amastigotes/1000 nucleated cells. The lines represent differences statistically significant for the asymptomatic dogs $(\mathrm{AD})$ in comparison to the symptomatic dogs. 

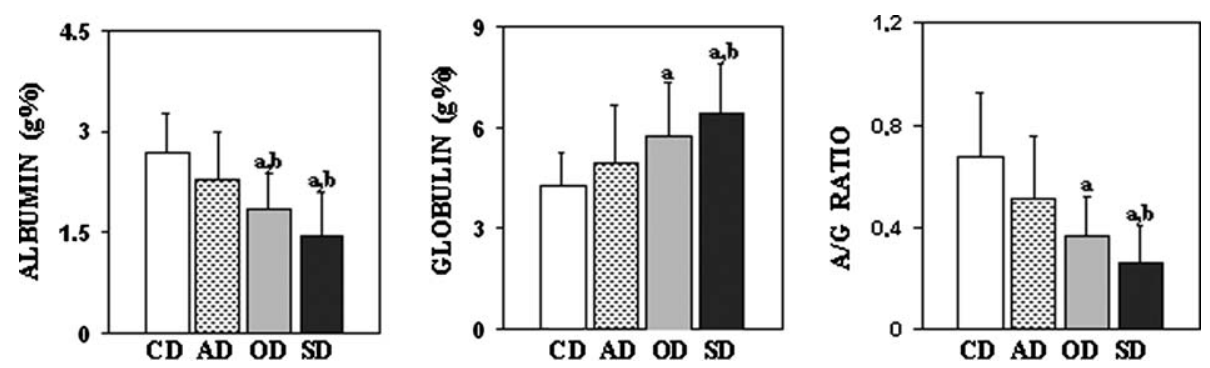

Fig. 3. Evaluation of biochemical parameters of naturally infected and non-infected dogs. The results are shown as average values \pm standard deviation. Control dogs (white bars); asymptomatic dogs (stained bars); oligosymptomatic dogs (gray bars) and symptomatic dogs (black bars). The letters "a" and "b" represent differences statistically significant for the control (CD) and asymptomatic dogs (AD), respectively.

lin concentration, when compared to control and asymptomatic animals. Those dogs from the oligosymptomatic group showed a significant decrease $(p<0.001)$ in $\mathrm{A} / \mathrm{G}$ ratio when compared to the control group, while the symptomatic animals presented a significant decrease $(p<0.001)$ in $\mathrm{A} / \mathrm{G}$ ratio in comparison to the control and asymptomatic groups (Fig. 3). However, only SD dogs presenting a positive correlation between both total protein and globulin values and IFAT titers $(r=0.6177 ; p=0.0108$; $r=0.5947 ; p=0.0151$, respectively).

The assessment of hematological parameters demonstrated severe anemia in the symptomatic animals, with a significant decrease $(p<0.001)$ in the number of erythrocytes, hemoglobin and hematocryt, in relation to the control, asymptomatic and oligosymptomatic groups. As for the white blood cells, statistical analysis showed significant difference among the symptomatic dogs and the other groups in the leukocytes series; however, no significant difference in absolute values of granulocytes neutrophils was observed among the groups of dogs under study. Statistical analysis revealed that the symptomatic dogs showed a significant decrease $(p<0.025)$ in leukocyte absolute values (leukopenia) when compared to the control group. Data analysis also demonstrated a significant decrease $(p<0.003)$ in absolute values of the eosinophylic granulocytes subpopulation from symptomatic dogs when compared to the control animals. The symptomatic group showed a significant decrease $(p<0.017)$ in absolute values of lymphocytes when compared to the control and asymptomatic groups. Data analysis showed also a significant decrease $(p<0.0017)$ in absolute values of monocytes for the symptomatic dogs when compared to control animals (Table 1).

\section{Discussion}

All clinical signs observed for the OD and SD groups have been extensively reported in the literature for both natural (Mancianti et al., 1988; Genaro et al., 1997) and experimentally infected dogs (Pinelli et al., 1994, 1995; Abranches et al., 1991). The high frequency of clinical signs presented by infected dogs allow us to observe that the disease gradually evolves from an apparently normal clinical picture, presented by $\mathrm{AD}$, to an active intermediate state, which includes some clinical manifestation (OD) that may evolve to a classical severe and terminal form of the disease (SD), characterized by a larger number of clinical signs. These findings suggested that the sub-clinical or asymptomatic disease may indicate that a good balance in parasite-host interaction is ongoing (Oliveira et al., 1993).

Hypergammaglobulinemia is a remarkable characteristic of CVL (Almeida et al., 2005). Several studies have reported a high antibody production during active $\mathrm{CVL}$,

Table 1

Evaluation of hematological parameters of naturally infected and non-infected dogs ${ }^{\dagger}$

\begin{tabular}{|c|c|c|c|c|}
\hline \multirow[t]{2}{*}{ Hematological parameters } & \multicolumn{4}{|c|}{ Clinical groups } \\
\hline & $\mathrm{CD}$ & $\mathrm{AD}$ & OD & SD \\
\hline Erythrocytes - million $/ \mathrm{mm}^{3}$ & $6.8 \pm 0.7$ & $5.8 \pm 0.7$ & $5.4 \pm 1.0$ & $4.2 \pm 1.2^{\mathrm{a}, \mathrm{b}, \mathrm{c}}$ \\
\hline Hemoglobin $-\mathrm{g} \%$ & $15.7 \pm 1.8$ & $14.0 \pm 2.3$ & $13.5 \pm 3.2$ & $9.8 \pm 2.8^{\mathrm{a}, \mathrm{b}, \mathrm{c}}$ \\
\hline Leukocytes $-10^{3} / \mathrm{mm}^{3}$ & $12.8 \pm 2.7$ & $14.1 \pm 5.0$ & $13.9 \pm 3.2$ & $10.2 \pm 3.9^{\mathrm{a}}$ \\
\hline Granulocytes & $9.1 \pm 3.1$ & $7.8 \pm 3.5$ & $9.4 \pm 3.4$ & $8.0 \pm 3.2$ \\
\hline Neutrophils & $7.2 \pm 3.1$ & $6.7 \pm 3.0$ & $8.5 \pm 3.2$ & $7.2 \pm 3.0$ \\
\hline Monocytes & $1.1 \pm 0.4$ & $1.0 \pm 0.5$ & $0.7 \pm 0.3$ & $0.5 \pm 0.4^{\mathrm{a}}$ \\
\hline
\end{tabular}

The letters "a", "b" and "c" represent statistically significant differences as compared to control (CD), asymptomatic (AD) and oligosymptomatic dogs (OD), respectively.

$\dagger$ The results are shown as the average values \pm standard deviation. 
with elevated levels of IgM and IgA circulating immunocomplexes (Margarito et al., 1998). Many reports have focused special attention to the search of alternative methodologies, as well as the use of specific antigen preparations, aiming to identify a better diagnostic method and prognostic tool for CVL clinical investigation. Some recombinant antigens have been pointed out as relevant candidates for CVL immunodiagnosis (Badaró et al., 1997; Soto et al., 1999; Rosário et al., 2005). Herein, the humoral immune response was evaluated by distinct immunological approaches, including IFAT, ELISA-extract and ELISA rK-39. Our data demonstrated that IFAT was able to identify as positive, all infected animals, despite their clinical status and have been used in this study as the gold standard to diagnosis CVL and select positive animals. ELISA-extract failed to detect IgG reactivity on two IFAT-seropositive animals, one AD and another OD. ELISA rK-39 was not able to detect IgG reactivity on five animals (three from AD and two from OD group). Genaro et al. (1997), using the recombinant protein rK-39 on a immunochromatographic assay (TRALd) to diagnosis CVL in an endemic area, demonstrated $92.1 \%$ of sensitivity and $99.5 \%$ of specificity, with significantly higher ability to detect Leishmania-specific antibodies when compared to IFAT. However, it was demonstrated that rK-39 was not able to predict the active infection when testing dog samples displaying antibody titers between 1:40 and 1:320, estimated by IFAT (Genaro et al., 1997). Our data are in agreement with these reports since all five seronegative animals detected by ELISA rK-39 displayed serological titles under $1: 320$ by IFAT. Together, our data demonstrated that ELISA rK-39 was able to confirm seropositivity in 35 out of 40 IFAT-positive animals ( $87.5 \%$ of sensitivity), re-enforcing previous reports of its applicability for CVL diagnosis (Rosário et al., 2005). The profiles of IgG antirK-39 presented by naturally infected dogs showed a positive correlation according to the clinical form of the disease. Similar results were also observed with IFAT and ELISA-extract. However, data analysis demonstrated that only IFAT titers was able to discriminate the mean IgG reactivity among infected dogs, with an enhancement of reactivity detected as more severe clinical status of infection was observed, with significant differences detected between $\mathrm{AD}$ and $\mathrm{SD}$ groups. According to Abranches et al. (1991), as the clinical signs appear, the antibody levels tend to be higher. However, Abranches et al. (1991) have not found a correlation between severity of the disease and total $\mathrm{IgG}$ titers. On the other hand, Pinelli et al. (1994) demonstrated that dogs experimentally infected with L. infantum, which also presented clinical signs, showed the highest antibody titers, similar to the results obtained by Genaro et al. (1988) in dogs naturally infected with $L$. infantum. Our data are in accordance with this later observation, since we found that SD group displayed the highest antibody titers in IFAT. Despite no significant differences on the mean antibody levels detected by ELISA-extract and ELISA rK-39 observed for SD, it was interesting to notice that a positive correlation between severity of disease and the antibody titers was detected by all methods.

Following infection, at the site of parasite inoculation, in the dog skin, the parasite disseminates carried out by histiocytes and Langerhans cells to the lymph node, bone marrow, spleen, liver, kidney, lungs and gastrointestinal tract, been rarely detected on the blood stream (Cardoso and Cabral, 1998). Infected animals presented hypertrophy of the Phagocytic Mononuclear System, leading to splenomegaly, hepatomegaly and generalized adenopathy (Lanotte et al., 1979). During Leishmania infection, tissue parasitism at different sites may differ and therefore is relevant for diagnosis purposes the identification of the major compartments presenting higher parasitism levels, in order to improve CVL diagnostic sensitivity. In this context, although the majority of authors consider the parasite search in the host tissues as the most reliable diagnostic method, its sensitivity and relative value have been questionable, considering the lack of uniformity of parasite distribution during natural infection. The parallel parasite search, at different affected tissues of the mammalian host organism, could contribute as a definitive criterion, which enables an investigator to choose the most appropriate biological specimen to be analyzed during diagnosis and prognosis practices. According to Cardoso and Cabral (1998), bone marrow biopsies are more sensitive than lymph nodes and therefore are more indicated for diagnosis purposes. Despite Ashford et al. (1995) reporting similar percentage of parasite detection in cultured versus impression bone marrow smear, our findings from $\mathrm{AD}$ and $\mathrm{SD}$ pointed out that bone marrow culture showed higher sensitivity as compared to bone marrow impression smears. Abranches et al. (1991) state that bone marrow examination led to positive results only during advanced stages of CVL. Our data confirmed such proposal as $88 \%$ of SD in contrast to $25 \%$ of $\mathrm{AD}$ showed positive results on bone marrow impression smear (Table 2). In general, our data demonstrated that parasitological survey performed on tissue impression smears (skin, spleen, liver and lymph node)

Table 2

Positivity of parasitological search on distinct tissues from dogs naturally infected with $\underline{\text { L. infantum }}^{\dagger}$

\begin{tabular}{llll}
\hline Tissues/methodology & \multicolumn{4}{l}{ Number of positive animals (\%) } \\
\cline { 2 - 4 } & \multicolumn{2}{l}{ Clinical groups } \\
\cline { 2 - 4 } & AD & OD & SD \\
\hline Bone marrow/culture & $6(50.0)$ & $6(50.0)$ & $15(94.0)^{\mathrm{a}, \mathrm{b}}$ \\
Bone marrow & $3(25.0)^{*}$ & $8(67.0)^{\mathrm{a}^{*}}$ & $14(88.0)^{\mathrm{a}}$ \\
Skin & $5(42.0)$ & $10(83.0)$ & $15(94.0)^{\mathrm{a}}$ \\
Spleen & $6(50.0)$ & $8(67.0)$ & $15(94.0)^{\mathrm{a}}$ \\
Liver & $5(42.0)$ & $8(67.0)$ & $14(88.0)^{\mathrm{a}}$ \\
Lymph node & $5(42.0)$ & $8(67.0)$ & $15(94.0)^{\mathrm{a}}$ \\
\hline
\end{tabular}

The letters "a" and "b" represent statistically significant differences as compared to AD and OD, respectively.

* Represents statistical differences within a given clinical group

$\dagger$ Results are expressed as number of positive dogs per group; in parenthesis, percentage of positive dogs per group. 
as well as cultured bone marrow proved to be more efficient to detect Leishmania organisms in AD than direct bone marrow impression smear, on which was possible to detect amastigotes only in $25 \%$ of the specimens analyzed (Table 2). These findings re-enforce that tissue parasitism intensity is parallel with the evolution of the clinical manifestations and point out the importance of the skin parasitism as a useful diagnostic tool when managing asymptomatic animals.

A great advance of parasitological methods is the possibility that such tests provide to evaluate the infection load through the parasite density (LDU). Thus, one may estimate the real parasite density and its contribution to the development of the pathology associated with the infection. Despite the relevance of quantitative parasite analysis for diagnosis and prognostic approaches there are no previous reports regarding the LDU index in natural or experimentally infected dogs. Our data demonstrated that parasite density in skin, bone marrow and spleen compartments were higher according to the severity of clinical manifestations (Fig. 2). Then, AD presented lower tissue parasitism as compared to SD on which LDU would count up to 7246, 1104 and 2564 amastigotes/1000-nucleated cells in skin, bone marrow and spleen sites, respectively. Interestingly, it was observed that all infected dogs, even those $\mathrm{AD}$, presented higher skin parasitism as compared to bone marrow. These findings on the skin LDU indexes reenforce the importance of cutaneous parasitism density to CVL diagnosis as well as the transmissibility of the infection from $\mathrm{AD}$ to susceptible species phlebotomine.

It is important to mention that these findings, regarding skin parasite load, described as LDU, is a pioneer report on canine visceral leishmaniasis. Moreover, the results of this current work highlight the importance quantitative investigations regarding the number of amastigote forms at different tissues. In addition, the assessment of parasite density would also contribute as therapeutic tool when measuring leishmanicidal activities of new promising drugs for CVL, regarding the several affected tissues.

Several biochemical and hematological alterations are observed in dogs natural or experimentally infected by $L$. infantum. A remarkable CVL characteristic is the dysproteinemia, with serum protein electrophoresis revealing decreased levels of albumin (A) and increased levels of globulin fraction $(G)$, leading to inversion of $A / G$ ratio (Keenan et al., 1984). Herein, we have confirmed these findings and also demonstrated that SD group presented lower $\mathrm{A} / \mathrm{G}$ ratio in comparison to $\mathrm{AD}$ and $\mathrm{CD}$, whereas OD differ only from CD (Fig. 3C). This inversion may occur due to decrease of albumin levels and increase of globulin, remarkably observed in SD. Further analysis demonstrated a positive correlation among both total protein and IFAT titers in the SD. These data revealed a relationship between the presences of exacerbated clinical forms of CVL, serum protein levels and humoral immune response. The factors that contribute to the enhancement of gammaglobulin levels in CVL are not yet completely understood, but this increase is probably due to the polyclonal activation B-lymphocytes triggered by $L$. infantum antigens as well as the synthesis of non-specific antibodies and the presence of circulating immunocomplexes (Marzochi et al., 1985; Margarito et al., 1998).

Regarding the hematological picture, it is common to observe normocytic/normochromic anemia, whereas, in the white blood cells, there is a leukopenia with moderated or accentuated lymphopenia (Abranches et al., 1991). The results presented in this study showed that during the symptomatic CVL decreased leukocyte counts, due to a drop in monocytes, eosinophils and mainly in lymphocyte population is one of the most relevant hematological finding. Leukopenia, associated to the symptomatic CVL, may be due a multifactorial mechanism on which medular dysfunction with diminished hematopoiesis, affected by an intense bone marrow parasitism as well as leukocyte recruitment and trapping into several organs are the major events (Alvar et al., 2004). It could also be related to secretion of suppressor cytokines triggered by Leishmania infection (Pinelli et al., 1994, 1995).

In conclusion, our study shows that the clinical evolution of CVL in naturally infected dogs promotes clear alterations in serological, parasitological and biochemical-hematological parameters. Since these alterations are directly correlated with CVL clinical status, they would be taken into account when dealing with diagnosis and prognosis features. The investigation of these laboratorial parameters, associated with the clinical aspect of CVL, is extremely important to be considered in routine clinical follow-up.

\section{Acknowledgments}

We are deeply in debt to Dr. GENARO, O., for his long support and enthusiasm to research on canine visceral leishmaniasis. He was also one of the most important investigators in the field of epidemiology, vaccines, and drugs against leishmaniasis with the focus on studies with dogs. He was and will always be remembered by his dedication to the progress of science. We also thank the people from Fundação Nacional da Saúde, Ministério da Saúde, Distrito Regional de Belo Horizonte, Minas Gerais, for their special dedication to this work. Finally, we grateful the Prof. Giovanni Gazzinelli by critical analysis of this paper. This work was supported by the $\mathrm{CNPq} / \mathrm{BR} / \mathrm{Grant}$ : 521124/98-0 and FAPEMIG/BR/Grant: CBS 2222/97.

\section{References}

Abranches, P., Silva-Pereira, M.C.D., Conceição-Silva, F., SantosGomes, G.M., Jans, J.G., 1991. Canine leishmaniasis: pathological and ecological factors influencing transmission of infection. J. Parasitol. 77, 561-577.

Almeida, M.A., Jesús, E.E., Sousa-Atta, M.L., Alves, L.C., Berne, M.E., Atta, A.M., 2005. Clinical and serological aspects of visceral leishmaniasis in northeast Brazilian dogs naturally infected with Leishmania chagasi. Vet. Parasitol. 127 (3-4), 227-232. 
Alvar, J., Cañavate, C., Molina, R., Moreno, J., Nieto, J., 2004. Canine Leishmaniasis. Adv. Parasitol. 57, 1-88.

Ashford, D.A., Bozza, M., Freire, M., Miranda, J.C., Sherlock, I., Euládio, C., Lopes, U., Fernades, O., Degrave, W., Barker Jr., R.H., Badaró, R., David, J.R., 1995. Comparison of the polymerase chain reaction and serology for the detection of canine leishmaniasis. Am. Soc. Trop. Med. Hyg. 58 (8), 251-255.

Badaró, R., Chun, P., Nakatani, M., Burns, J., Skeiky, Y., Houghton, R.L., Arias, J., Monteiro, P., Genaro, O., Reed, S.G., 1997. TRALd a rapid test specific for serodianosis of visceral leishmaniasis. Act. Parasitol. Tur. 21 (1), 175.

Camargo, M.E., Rebonato, C., 1969. Cross-reactivity in fluorescence tests for Trypanosoma and Leishmania antibodies. A simple inhibition procedure to ensure specific results. Am. J. Trop. Med. Hyg. 18, 500-505.

Cardoso, L., Cabral, M., 1998. Leishmania and canine Leishmaniasis. Rev. Port. C. Vet. XCIII (527), 122-141.

Costa, C.A., Genaro, O., Lana, M., Magalhães, P.A., Dias, M., Michalick, M.S., Melo, M.N., Costa, R.T., Magalhães-Rocha, M.N., Mayrink, W., 1991. Leishmaniose visceral canina: avaliação da metodologia sorológica utilizada em inquéritos epidemiológicos. Rev. Soc. Med. Trop. 24, 21-25.

Dace, J., Lewis, S., 1984. Practical Hematology, sixth ed. Churchil Livingstone, London, $453 \mathrm{pp}$.

Dias, D.V., Da Costa, C.A., Toledo, V.P.C.P., Bambirra, E., Genaro, O., Michalick, M.S.M., Costa, R.T., Mayrink, W., Oréfice, F., 1999. Leishmaniose visceral canina - Estudo parasitológico e histológico em olhos de cães - Parte I. Rev. Bras. Oftal. 58 (5), 331-337.

Genaro, O., Costa, R.T., França-Silva, J.C., Reis, A.B., Silva, J.C., Vieira, E.P., Arias, J.R., Monteiro, P.S., Reed, S.G., Mayrink, W., Costa, C.A., Netto, E.M., Badaró, R., 1997. Evaluation of an immunochromatographic assay for the diagnosis of dogs experimentally and naturally infected with Leishmania chagasi, in Brasil. Acta Parasitol. Turc. 21 (1).

Genaro, O., Mayrink, W., Michalick, M.S.M., Dias, M., Da Costa, C.A., Melo, M.N., 1988. Naturally occurring visceral leishmaniasis in dogs: clinical aspects. Mem. Inst. Oswaldo Cruz. 83, 43.

Grimaldi Jr., G., Tesh, R.B., 1993. Leismanioses of the new world: current concepts and implications for future research. Clin. Microbiol. Ver. 6 (3), 230-250.

Keenan, C.M., Hendricks, L.D., Lightner, L., Webster, H.K., Johnson, A.J., 1984. Visceral leishmaniasis in the German Shepherd dog-I Infection, clinical disease, and clinical pathology. Vet. Pathol. 21, 74-79.

Lanotte, G., Rioux, J.A., Perieres, J., Vollhardt, Y., 1979. Écologie des leishmanioses dans le sud de la France. 10. Les forms évolutives de la leishmanioses viscérale canine. Elaboration d'unetypologie bio-clinique à finalité é epidemiémiologique. Ann. Parasitol. 54, 277-295.

Lowry, O.H., Rosebrough, N.J., Farr, A.L., Randall, R.J., 1951. Protein measurement with the folin phenol reagent. J. Biol. Chem. 193, 263-275.

Mancianti, F., Gramiccia, M., Gradoni, L., Pieri, S., 1988. Studies on canine leishmaniais control. I. Evolution of infection of different clinical forms of canine leishmaniasis following antimonial treatment. Trans. R. Soc. Trop. Med. Hyg. 82, 566-567.

Mancianti, F., Falcone, M.L., Giannelli, C., Poli, A., 1995. Comparison between an enzyme-linked immunosorbent assay using a detergentsoluble Leishmania infantum antigen and indirect immunofluorescence for the diagnosis of canine leishmaniasis. Vet. Parasitol. 59, 13-21.

Margarito, J.M., Lucena, R., Lopez, R., Molleda, J.M., Martin, E., Ginel, P.J., 1998. Levels of IgM and IgA circulating immune complexes in dogs with leishmaniasis. Zent. Vet. 45 (5), 263-267.

Marzochi, M.C.A., Coutinho, S.G., Souza, W.J.S., Toledo, L.M., Grimald Jr., Momen, H., Pacheco, R.S., Sabroza, P.C., Souza, M.A., Rangel Jr., F.B., Tramontano, N., 1985. Canine visceral leishmaniasis in Rio de Janeiro, Brazil. Clinical, parasitological, therapeutically and epidemiological findings (1977-1983). Mem. Inst. Oswaldo Cruz. 80, 349-357.

Molina, R., Amela, C., Nieto, J., San-Andres, M., Gonzalez, F., Castillo, J.A., Lucientes, J., Alvar, J., 1994. Infectivity of dogs naturally infected with Leishmania infantum to colonized Phlebotomus perniciosus. Trans. Roy. Soc. Trop. Med. Hyg. 88, 491-493.

Oliveira, G.G.S., Santoro, F., Sadigursky, M., 1993. The subclinical form of experimental visceral leishmaniasis in dogs. Mem. Inst. Oswaldo Cruz. 88 (2), 243-248

Pinelli, E., Killick-Kendrick, R., Wagenaar, J., Bernadina, W., Real, G., Ruitenberg, J., 1994. Cellular and humoral immune responses in dogs experimentally and naturally infected with Leishmania infantum. Infect. Immunol. 62 (1), 229-235.

Pinelli, E., Gonzalo, R.M., Boog, C.J.P., Rutten, V.P.M.G., Gebhard, D., Del Real, G., Ruitenberg, E.J., 1995. . Leishmania infantum-specific T cell lines derived from asymptomatic dogs that lyse infected macrophages in a major histocompatibility complex-restricted manner. Eur. J. Immunol. 25, 1594-1600.

Rosário, E.Y., Genaro, O., França-Silva, J.C., da Costa, R.T., Mayrink, W., Reis, A.B., Carneiro, M., 2005. Evaluation of enzyme-linked immunosorbent assay using crude Leishmania and recombinant antigens as a diagnostic marker for canine visceral leishmaniasis. Mem. Inst. Oswaldo Cruz. 100 (2), 197-203.

Soto, M., Requena, J.M., Quijada, L., Alonso, C., 1999. Antigenicity of the Leishmania infantum histones $\mathrm{H} 2 \mathrm{~B}$ and $\mathrm{H} 4$ during canine viscerocutaneous leishmaniasis. Clin. Exp. Immunol. 115 (2), 342-349.

Stauber, L.A., 1955. Leishmaniasis in the hamster. In: Cole, W.H. (Ed.), Some Physiological Aspects and Consequence of Parasitism. Rugers University Press, New Brunswick, NJ, pp. 77-90.

Strauss-Ayali, D., Baneth, G., 2001. Canine visceral leishmaniasis. In: Carmichael (Ed.), Recent Advances in Canine Infectious Diseases. IVIS, Ithaca, NY.

Tesh, R.B., 1995. Control of zoonotic visceral leishmaniasis: is it time to change strategies? Am. J. Trop. Med. Hyg. 52 (3), 287-292.

Vieira, J.B., Coelho, G.E., 1998. Leishmaniose visceral ou calazar: aspectos epidemiológicos e de controle. Rev. Soc. Bras. Med. Trop. I (II), 85-92. 